\title{
Analytical approach of an FSO Link with alamouti- type STBC considering weak atmospheric turbulence
}

\author{
M. S. Islam ${ }^{1}$, M. Morshed ${ }^{1}$, R. C. Roy ${ }^{2}$ and M. H. A. Begum ${ }^{3 *}$ \\ ${ }^{I}$ Department of Electrical and Electronic Engineering, Ahsanullah University of Science and Technology, Dhaka, Bangladesh \\ ${ }^{2}$ Pilot Plant and Process Development Center (PP \& PDC), BCSIR, Dhaka, Bangladesh \\ ${ }^{3}$ BCSIR Laboratories, Dhaka, BCSIR, Dhaka, Bangladesh
}

Received: 23 October 2017

Revised: 02 January 2018

Accepted: 31 January 2018

DOI: http://dx.doi.org/10.3329/bjsir.v53i3.38267

\begin{abstract}
An analytical approach is proposed to evaluate the bit error rate (BER) performance of a direct detection on-off keying (OOK) free space optical link over log-normal atmospheric turbulence-induced fading channels based on modified Alamouti code presented by Simon and Vilnrotter and maximal ratio combining (MRC) at the receiver. Simulations show that due to coding and diversity at the receiver, a significant improvement in the BER performance is obtained at a given BER. For diversity order of 2 in both transmitter and in receiver, the amount of BER improvement is $6.6 \mathrm{~dB}$ at BER $10^{-5}$. Further increment of the receiver diversity from 2 to 4 gives additional $3 \mathrm{~dB}$ improvement at the same BER. A drastic improvement of the BER performance is also possible for increasing number of receiver diversity which may increase hardware complexity. Simulation results are demonstrated to confirm the analytical results.
\end{abstract}

Keywords: Bit error rate; Space-time block code (STBC); Free space optic: Maximal ratio combining

\section{Introduction}

Free space optical (FSO) communication is a very promising and cost effecting technology for the future optical communication networks (Andrews et al., 2001; Arnon, 2003; Vincent, 2006). A major impairment in FSO links is the atmospheric turbulence-induced fading which causes fluctuations in both the intensity and phase of the received light signal which results in impairments of the link performance (Antonio, 2007; Jindal and Lozano, 2010; Bhuiyan et al., 2010; Tarokh et al., 1999; Huang et al., 1993; Zhu and Kahn, 2002). For free space optical transmission systems, most frequently used system is Intensity Modulated Direct Detection (IM/DD) system. In such system, the intensity of an optical source is modulated to transmit signals. For FSO systems, although the power efficiency is inferior to pulse position modulation (PPM), OOK encoding is more commonly used due to its efficient bandwidth usage and robustness to timing errors. Therefore, in this research work, for FSO systems we prefer intensity modulation/direct detection (IM/DD) with an OOK technique.

For the Microwave wireless communication it is found that Alamouti coding can significantly mitigate the signal fading by increasing the diversity gain (Alamouti 1998; Navidpour et al., 2007; Stephen et al., 2005) where complex valued vectors were essential for adding the diversity. Simon et al. have proposed a modified version of Alamouti code for FSO intensity modulated direct detection (FSO IM/DD) links. In Alamouti code and other orthogonal STBCs the signal polarity (phase) could be detected at the receiver (Simon and Vilnrotter, 2005). This is not possible with unipolar modulations such as OOK (on-off keying) and PPM (pulse position modulation) commonly used in FSO IM/DD links. 
Simon et al. have overcome this problem by modifying Alamouti code in a clever manner to avoid the necessity of transmitting the negative of a modulated signal, thereby allowing the use of unipolar modulation techniques. This coding scheme is in fact more adaptable for implementation in the free space optical channel due to diversity adding capability on real valued data (Antonio, 2007; Simon and Vilnrotter, 2005). A turbulent atmospheric channel causes the intensity of an optical beam travelling through it to fluctuate randomly due to random changes in refractive index along the beam's path. Moreover in the FSO communication system, increasing the length of the transmission path is also very much challenging due to the impact of strong atmospheric turbulence. The different types of noise encountered during optical detection were discussed with the view to understanding the limit imposed on the system performance by each of them. The review work revealed that the transmitted photons obey the Poisson distribution and this can be adequately approximated as the tractable Gaussian distribution for large photoelectron counts as is the case in terrestrial FSO systems.

\section{Materials and methods}

\section{System model}

The overall system model for FSO system is depicted in Fig. 1. The Alamouti-type STBC coded OOK data is transmitted by the laser sources and received by photo detector using direct detection method. At the receiver end, the incoming optical radiation is passed through an optical band pass filter (OBPF) before being converted into an electrical signal by the photo detector. The OBPF is used to limit the amount of background radiation noise detected by the photo detector. The received signals (photo detectors current) are then passed through the pre-amplifier (PA). The outputs of the pre-amplifiers are then put into the combiner. Various methods such as maximal ratio combiner (MRC), selection combiner (SC), equal gain combiner (EGC) etc have been proposed for combining independently faded signal components and the trade off among these methods is the receiver complexity versus transmission performance improvement. In FSO system, all the signals are the light intensity, they must be real signals. For OOK modulation, the signals can be described as:

$$
\begin{array}{lll}
s_{1}=0 ; & 0<\mathrm{t} \leq \mathrm{T}_{\mathrm{b}} \quad \text { light off } \\
s_{2}=A ; & 0<\mathrm{t} \leq \mathrm{T}_{\mathrm{b}} \quad \text { light on }
\end{array}
$$

where ' $A$ ' is a positive constant related to the intensity of the light source and $T_{b}$ is the bit duration. We can express one of these signal waveforms in terms of the other by

$$
s_{i}=-s_{j}+A \quad ; j \neq i
$$

The new formation of the transmit symbols become as:

$$
\left\{s_{1}, s_{2}\right\} \rightarrow\left[\begin{array}{ll}
x_{1} & \bar{x}_{2} \\
x_{2} & x_{1}
\end{array}\right]
$$

For the FSO system we define the complement of a signal $x_{i}$ as $x_{i}$ where $x_{i}$ and $x_{i}$ are used for "light on" and "light off" respectively. For example, if, $x_{i}$ then

$$
\bar{x}_{i}=s_{2}=-s_{1}+A=-x_{i}+A
$$

Otherwise, if $x_{i}=s_{2}$, then $x_{i}=s_{1}=-s_{2}+A=-x_{i}+A$

\section{Channel model}

In homogeneities caused by turbulence can be viewed as discrete cells, or eddies of different temperature, acting like refractive prisms of different sizes and indices of refraction. The interaction between the laser beam and the turbulent medium results in random phase and amplitude variations (scintillation) of the information-bearing optical beam which ultimately results in performance degradation of FSO links.

Atmospheric turbulence results in random fluctuation of the atmospheric refractive index, $n$ along the path of the optical field/radiation traversing the atmosphere. This refractive index fluctuation is the direct end product of random 
variations in atmospheric temperature from point to point (Pratt, 1969). These random temperature changes are a function of the atmospheric pressure, altitude and wind speed.

The atmospheric turbulence impairs the performance of an FSO link by causing the received optical signal to vary randomly thus giving rise to signal fading. The fading strength depends on the link length, the wavelength of the optical radiation and the refractive index structure parameter of the $C^{2}$ channel.

The log-normal distribution is generally used to model the fading associated with the weak atmospheric turbulence regime. This model is mathematically tractable and it is characterized by the Ryotov variance $\sigma^{2}$. The turbulence induced fading is termed weak when $\sigma^{2}<1.2$ and this defines the limit of validity of the log-normal model. Beyond the weak turbulence regime, other models such as the gamma-gamma and the negative exponential will have to be considered. The Ryotov variance $\sigma^{2}$ can be calculated as

$$
\sigma_{X}^{2}=1.23 C_{n}^{2}\left(\sqrt[6]{k^{7} L^{11}}\right)
$$

where $L$ is the propagation distance and $k$ is the wave number.

The amplitude of the random path gain, $H=e^{x}$, where $X$ is normal with mean $\mu_{X}$ and variance $\sigma^{2}$. Thus, the logarithm of the field amplitude scale factor is normally distributed. The optical intensity $I=H^{2}$ is also $\log$ normally distributed in this case (Stephen et al., 2005, 2005a). The pdf for $H$ is

$$
f_{H}(a)=\frac{1}{\left(2 \pi \sigma_{X}^{2}\right)^{0.5} a} \exp \left(-\frac{\left(\log _{e} a-\mu_{x}\right)^{2}}{2 \sigma_{X}^{2}}\right), \quad a>1
$$

So that the mean path intensity is unity, i.e., $E\left[H^{2}\right]=1$. For the log-normal distribution, the scintillation index, defined as

$$
\text { S.I. }=\frac{E\left[H^{4}\right]}{E^{2}\left[H^{2}\right]}-1
$$

This index can be related to the parameter $\sigma^{2}$ by S.I. $=e^{402}-1$. Typical values appearing in the literature for S.I. are in the range $0.4-1.0$.

\section{Ber analysis}

The transmitted optical signal by the lasers-

$$
\text { for laser-1, } \quad \begin{aligned}
S_{1}(t) & =\sqrt{2 P_{s} \varepsilon} \quad \text { for } \quad 0 \angle t \angle \frac{T_{b}}{2} \\
& =-\sqrt{2 P_{s} \varepsilon}+A \text { for } \quad \frac{T_{b}}{2} \angle t \angle T_{b}
\end{aligned}
$$

$$
\text { for laser-1, } \begin{array}{rlrl}
S_{2}(t) & =\sqrt{2 P_{s} \varepsilon} & \text { for } & 0 \angle t \angle \frac{T_{b}}{2} \\
& =\sqrt{2 P_{s} \varepsilon} \quad \text { for } & \frac{T_{b}}{2} \angle t \angle T_{b}
\end{array}
$$

where $T_{b}$ is the bit duration.

At the receiver end, the received photo current for $i^{\text {th }}$ photodetector is -

$i_{i}(t)=R_{d}\left|S_{i}(t)\right|^{2} \times\left|h_{i}\right|^{2}+n(t)=2 R_{d} P_{s} \varepsilon\left|h_{i}\right|^{2}+n(t)=2 R_{d} I\left|h_{i}\right|^{2}+n(t)$

The total photodetector's current is given by

$$
\begin{gathered}
i(t)=\left[\begin{array}{ll}
\left|h_{11}\right|^{2} & \left|h_{21}\right|^{2} \\
\left|h_{12}\right|^{2} & \left|h_{22}\right|^{2}
\end{array}\right] \times 2 R_{d} I+n(t) \\
i_{j i}(t)=\left|h_{j i}\right|^{2} \times 2 R_{d} I+n(t) \\
i(t)=\sum_{j=1}^{L} \sum_{i=1}^{M} i_{j i}
\end{gathered}
$$

where,

$R_{d}=$ photodetector responsivity

$P_{s}=$ symbol power (power at the bias level of the semiconductor)

$$
\begin{aligned}
& \varepsilon=\text { modulation index } \\
& I=\text { maximum optical intensity } \\
& n(t)=\text { AWGN (Additive White Gaussian Noise) } \\
& \quad=\text { Thermal Noise }+ \text { Background radiation shot Noise }
\end{aligned}
$$

The additive white Gaussian noise $n(t)$ consists of both the thermal noise and the background radiation shot noise.

The shot noise is,

$$
\sigma_{\text {shot }}^{2}=2 q B . i_{\text {sho }}
$$

And the background noise is

$$
\sigma_{b g}^{2}=2 q B_{e l .} i_{b g}
$$

where ,

$B_{e l}=$ Post detection electrical filter bandwidth.

$$
i_{b g}=\text { Background current }=R_{d} I_{b g} ;
$$

Considering $I_{b g}=$ background radiation irradiance. Now, equation (9) can be written as-

$$
\sigma_{b g}^{2}=2 q B_{e l .} R_{d} I_{B g}
$$


Here the thermal noise

$$
\sigma_{t h}^{2}=4 k T B_{e l} / R_{L}
$$

where,

$$
\begin{aligned}
& k=\text { Boltzman constant }=1.38 * 10^{-23} \\
& R_{L}=\text { Receiver circuit load resistance } \\
& T=\text { Temperature }
\end{aligned}
$$

Now, considering the background noise and thermal noise power from equations (9) and (10), total noise power is-

$$
\begin{aligned}
& \sigma^{2}=\sigma_{b g}^{2}+\sigma_{t h}^{2} \\
= & 2 q B_{e l .} R_{d B g} I_{B g}+4 k T B_{e l} / R_{L} \\
= & 2 B_{e l .}\left(q R_{d B g} I_{B g}+2 k T / R_{L}\right)
\end{aligned}
$$

In presence of turbulence the total bit energy to noise spectral density ratio is expressed as

$$
\begin{aligned}
\bar{\gamma} & =\bar{h}_{j i}^{2} E_{b} / N_{0} \\
& =\bar{h}_{j i}^{2} 2 R_{d} P_{s} \varepsilon T_{b} / N_{0} \\
& =\bar{h}_{j i}^{2} 2 R_{d} P_{s} \varepsilon /\left(N_{0} \frac{1}{T_{b}}\right) \\
& =\bar{h}_{j i}^{2} 2 R_{d} P_{s} \varepsilon / \sigma^{2}
\end{aligned}
$$

In OOK, $E_{b}$ represent the energy of the detected pulse in the 'on' state.

Assuming transmission matrices based on real orthogonal designs for full rate and a full diversity space time block code, the adoption of an array based on $M$ laser sources, all pointed towards a distant array of $N$ photodetectors, implies a diversity of order $N M$, so that the conditional BER is given by (Huang et al., 1993),

$$
P_{b}\left(E \mid\left\{h_{j i}\right\}_{\substack{1 \leq i \leq M \\ 1 \leq j \leq N}}\right)=Q\left(\sqrt{\frac{E_{b}}{2 N_{0}} \sum_{j=1}^{N} \sum_{i=1}^{M} h_{j i}^{2}}\right)
$$

where $Q(x)$ is the Gaussian- $Q$ function with argument $x$.

From equation (13), using the alternating form of $\mathrm{Q}(\mathrm{x})$, is obtained as

$$
P_{b}\left(E \mid h_{j i}\right)=\frac{1}{\pi} \int_{0}^{\frac{\pi}{2}} \prod_{j=1}^{N} \prod_{i=1}^{M} \exp \left(-\frac{\bar{\gamma} h_{j i}^{2}}{2 \times 2 \sin ^{2} \theta}\right) d \theta
$$

The conditional BER averaged over the pdf of the channel irradiance thus yielding

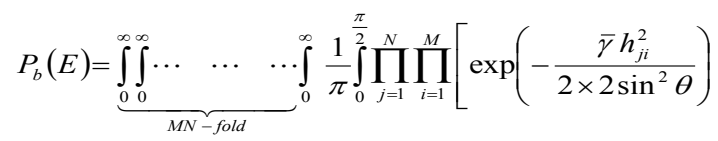

where

$$
p\left(h_{j i}\right)=\frac{1}{\left(2 \pi \sigma_{X}^{2}\right)^{0.5} h_{j i}} \exp \left(-\frac{\left(\log _{e} h_{j i}-\mu_{x}\right)^{2}}{2 \sigma_{X}^{2}}\right), \quad a>1
$$

where the mean . $\mu_{x}=-\frac{\sigma_{x}^{2}}{2} \quad$ By changing the order of integration in equation (15) and one gets subsequently grouping the terms of index ij,

where

$$
P_{b}(E)=\frac{1}{\pi} \int_{0}^{\frac{\pi}{2}}[D(\theta, \bar{\gamma})]^{N M} d \theta
$$

$$
D(\theta, \bar{\gamma})=\int_{0}^{\infty} \exp \left(-\frac{\bar{\gamma} h^{2}}{2 \times 2 \sin ^{2} \theta}\right) \times \frac{1}{\sqrt{2 \pi \sigma_{x}^{2}} h} \exp \left(-\frac{\left(\log _{e} h+\frac{\sigma_{x}^{2}}{2}\right)^{2}}{2 \sigma_{x}^{2}}\right) d h
$$

A closed-form solution of equation (16) does not exist and could result in truncating the upper limit using the numerical integration. By using an alternative representation of the Q-function together with the Gauss-Hermite quadrature integration (Popoola et al., 2008), these problems can be circumvented. These are expressed as

$$
\begin{gathered}
Q(x)=\frac{1}{\pi} \int_{0}^{\frac{\pi}{2}} \exp \left(-\frac{x^{2}}{2 \sin ^{2} \theta}\right) d \theta \\
\int_{-\infty}^{\infty} f(x) \exp \left(-x^{2}\right) d x \cong \sum_{i=1}^{m} w_{i} f\left(x_{i}\right)
\end{gathered}
$$

where and represent the zeros and weight factors of the $m$ th order Hermite polynomial respectively (Popoola et al., 2008; Ghassemlooy et al., 2009). The degree of accuracy of equation (17) is determined by the value of $\mathrm{m}$.

$$
\text { Let us consider, } x=\frac{\left(\log _{e} h+\frac{\sigma_{x}^{2}}{2}\right)}{\sqrt{2 \sigma_{x}^{2}}}
$$

Thus, $\quad h=\exp \left(\sqrt{2 \sigma_{x}^{2}} x-\frac{\sigma_{x}^{2}}{2}\right)$ and

$$
d h=\sqrt{2 \sigma_{x}^{2}} \exp \left(\sqrt{2 \sigma_{x}^{2}} x-\frac{\sigma_{x}^{2}}{2}\right) d x
$$


Using equations (17), (18) and (19) in equation (16) the resultant BER is obtained as,

$$
P_{b}(E)=\frac{1}{\pi} \int_{0}^{\frac{\pi}{2}}\left[\frac{1}{\sqrt{\pi}} \sum_{i=1}^{m} w_{i} \exp \left(-\frac{\bar{\gamma} \exp \left(2 \sqrt{2 \sigma_{x}^{2}} x_{i}-\sigma_{x}^{2}\right)}{2 \times 2 \sin ^{2} \theta}\right)\right]^{M N} d \theta
$$

\section{Results and discussion}

Following the aforementioned analytical approach, the BER performance of an IM/DD optical FS link with Alamouti-type STBC over log normal turbulence channel is evaluated

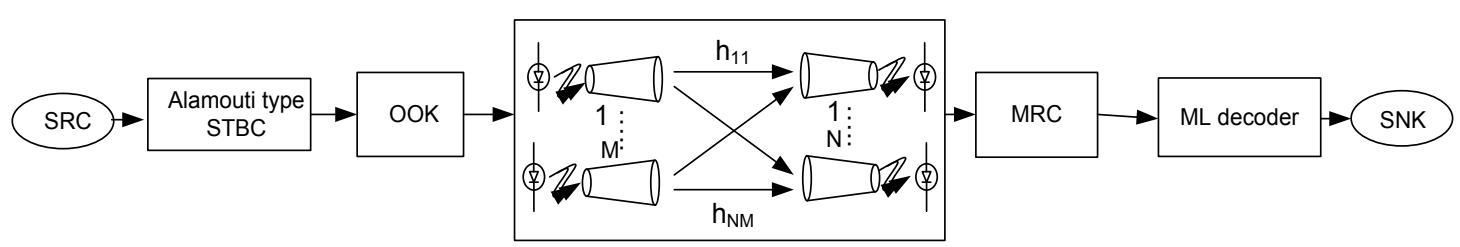

Fig.1. System model of an FSO link with STBC

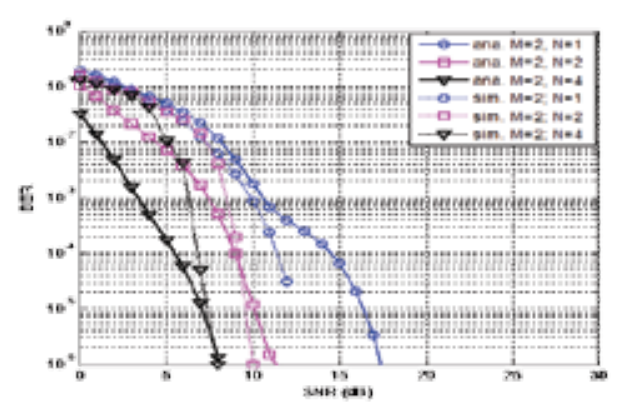

Fig. 2. Analytical and Simulated BER performance of an FSO link over log-normal turbulence model with Alamouti type STBC with different receiver diversity.

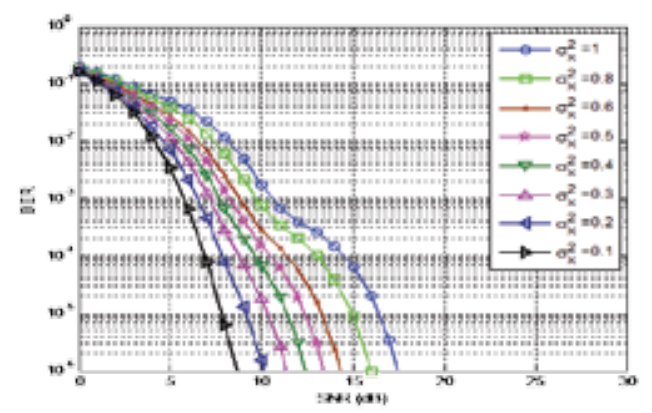

Fig. 3. BER performance curve for FSO link over lognormal turbulent condition with varying turbulence variance considering $M=2, L=1$ and $M R C$ at the receiver.

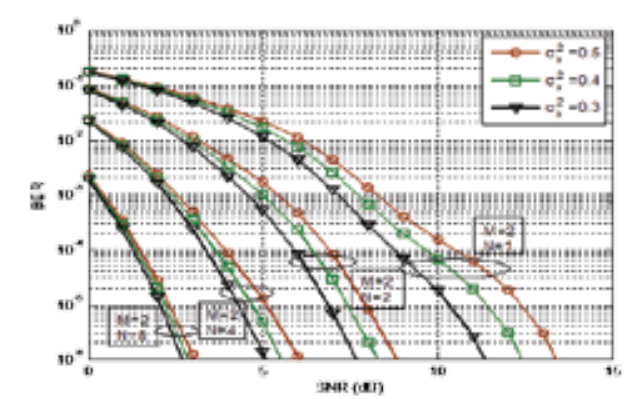

Fig. 4. BER performance curve for FSO log-normal with different detector diversity with different variance using MRC at the receiver. numerically. Results are evaluated in terms of BER as a function of average SNR with Alamouti-type STBC considering weak turbulence model. Figure 2 shows both analytical and simulated results. The amount of BER improvement for $(2 \times 2)$ and $(2 \times 4)$ STBC is of the order $6.6 \mathrm{~dB}$ and $9.6 \mathrm{~dB}$ respectively w.r.t $(2 \times 1)$ at BER $10^{-5}$. In figure 3 it is clearly shown that with the increasing turbulence variance the BER performance falls significantly. Figure 4 shows the comparison of the BER performances due to increment of number of detectors along with the variation of the turbulence variance. At turbulence variance 0.3 , the BER improvement is $4.5 \mathrm{~dB}$, $7 \mathrm{~dB}$ and $9.5 \mathrm{~dB}$ for photodetector 2,4 and 8 respectively at BER $10^{-6}$. The more the number of detectors the better the link performance is.

\section{Conclusion}

The error rate performance for modified Alamouti-type STBC in FSO communication systems with direct detection operating over log normal turbulence channels is analyzed in this paper. The proposed analytical approach almost matches with the simulation results. We have simulated the performance of an optical link using Alamouti type space-time block coding at weak atmospheric turbulent condition considering different receiver diversity and also compare the BER curves. In this paper, it is clearly found that Alamouti-type STBC code can significantly improve the BER performance of FSO system with OOK technique considering weak atmospheric turbulent condition. 


\section{References}

Antonio GZ (2007), Error Rate Performance for STBC in Free-Space Optical Communications through Strong Atmospheric Turbulence, IEEE Comm. Letters, Vol. 11, no. 5, pp 390-392.

Andrews L, Phillips R, and Hopen C (2001), Laser Beam Scintillation with Applications. SPIE Press.

Arnon S (2003), Optimization of Urban Optical Wireless Communication Systems, IEEE Transactions on Wireless Communications 4(2): 626-629. DOI: 10.1109/TWC.2003.814351

Alamouti SM (1998), A Simple Transmit Diversity Technique for Wireless Communications, IEEE Journal on selected areas on Communications 16(8): 1451-1458.

Bhuiyan TA, Hassan MZ, Tanzil SMS, Hayder and Majumder SSP (2010), Performance Improvement of IM-DD Free Space Optical CDMA (Attenuated by Strong Atmospheric Turbulence) with Maximal Ratio Combining, Proc. CICN '10 Computational Intelligence and Communication Networks Conf., 2010 IEEE Computer Society, Washington DC, USA, ISBN: 978-0-7695-4254-6, DOI-10.1109/CICN.141.

Ghassemlooy Z, Popoola WO, Ahmadi V and Leitgeb (2009), MIMO Free-Space Optical Communication Employing Subcarrier Intensity Modulation in Atmospheric Turbulence Channels, Communications Infrastructure, Systems and Applications in Europe, Springer-Verlag Berlin Heidelberg, Vol. 16. pp 61-74, ISBN 978-3-642-11283-6, DOI: 10.1007/978-3-64211284-3_7.

Huang WJ. Takayagi JT. Sakanaka T and Nakagawa M (1993), Atmospheric Optical Communication system Using Subcarrier PSK Modulation, IEEE International Conference, Vol. 3, pp 1597-1601.

Jindal N and Lozano A (2010), De-hyping Transmit Diversity in Modern MIMO Cellular Systems, Proc. $28^{\text {th }}$ Global telecommunications - GLOBECOM2009 IEEE conf. Honolulu, HI, USA, pp 1-6.
Navidpour SM, Uysaland M and Kavehrad M (2007), BER Performance of Free-Space Optical Transmission with Spatial Diversity, IEEE Transactions on Wireless Communications 6(8): 2813-2819.

Pratt WK (1969), Laser Communication Systems, $1^{\text {st }}$ Ed. John Wiley \& Sons, Inc., New York.

Popoola WO, Ghassemlooy Z, Allen JIH, E. Leitgeb and Gao S (2008), Free-space optical communication employing subcarrier modulation and spatial diversity in atmospheric turbulence channel, IET Optoelectronic 2: 16-23.

Simon M and Vilnrotter V (2005), Alamouti-type space-time coding for freespace optical communication with direct detection, IEEE Trans. Wireless Commun. 4(1): 35-39. DOI: 10.1109/TWC.2004.840216

Stephen WG, Maïté B, Qianling C and James H (2005), Free-Space Optical MIMO Transmission with Q-ary PPM, IEEE Trans. on Communications 53(8): pp 1402-1412.

Stephen WG, Maïté B, Qianling C and James H (2005a), Optical Repetition MIMO Transmission With Multipulse PPM, IEEE journal on selected areas in communications 23(9): 1901-1910. DOI: 10.1109/JSAC.2005.853804

Tarokh V, Jafarkhani H and Calderbank AR (1999), Space-time block codes from orthogonal designs, IEEE Trans. Inf. Theory 45(5): 1456-1467. DOI: $10.1109 / 18.771146$

Vincent SWC (2006), Free-Space Optical Communication, IEEE, Fellow, OSA Journal of Light wave Technology 24(12): 4750-4762.

Zhu X and Kahn JM (2002), Free-space optical communication through atmospheric turbulence channels, IEEE Trans. Commun. 50(8): 1293-1300. DOI: 10.1109/TCOMM.2002.800829 